\title{
Multimodal Imaging in Acute and Chronic Central Serous Chorioretinopathy
}

\author{
An Vo, OD \\ Assistant Professor \\ Western University \\ of Health Sciences College \\ of Optometry \\ Jessica Haynes, OD, FAAO \\ Associate Optometrist \\ Charles Retina Institute; \\ Southern College \\ of Optometry \\ Adjunct Faculty
}

\begin{abstract}
Numerous theories have been proposed regarding the pathophysiology of central serous chorioretinopathy (CSR), with no consensus on its exact etiology. Research has led to a variety of treatment possibilities that target specific mechanisms. Multimodal imaging, including fundus auto-fluorescence (FAF), optic coherence tomography (OCT), fluorescein angiography, and indocyanine green angiography, identifies vascular and structural alterations, which can confirm the diagnosis of CSR and effectively tailor treatment to help maximize the visual prognosis in these patients. Imaging characteristics of CSR and case examples will be explored.
\end{abstract}

\section{KEY WORDS:}

Central serous retinopathy, central serous chorioretinopathy, CSR, CSCR

\section{INTRODUCTION}

Central serous retinopathy is a relatively common condition characterized by localized areas of neurosensory retinal detachment, most often in the macula, with or without detachment of the retinal pigment epithelium (RPE). ${ }^{1}$ Patients range in presentation, with common symptoms including mild blur, metamorphopsia, dyschromatopsia, and reduced contrast sensitivity. CSR is typically unilateral but may present bilaterally, though almost exclusively asymmetrically. ${ }^{2}$

CSR is the fourth-most common vision-threatening disease worldwide after macular degeneration, diabetic retinopathy, and retinal vein occlusion. ${ }^{3,4}$ In males, the average incidence worldwide is 10 per 100,000, and males are six times more likely to be affected than females. There is a slight racial predilection among Asians and Caucasians compared to those of African background, although CSR in African-Americans has been associated with poorer visual outcome and more aggressive presentation., ${ }^{2,4}$ Mean age at diagnosis ranges between 39 and 51 years, with patients older than 50 at higher risk for choroidal neovascularization and a prolonged course. ${ }^{4}$

In acute cases, CSR often resolves without intervention. Several treatment options have been proposed for chronic cases, including focal laser photocoagulation, photodynamic therapy (PDT), anti-vascular endothelial growth factor (anti-VEGF) injection, anti-corticosteroid therapy, aldosterone inhibitors such as eplerenone, and discontinuation of systemic steroid use. ${ }^{5}$

The efficacy of treatment options such as anti-VEGF, anti-corticosteroid therapy, and aldosterone inhibitors remains controversial. Additionally, not all presentations of CSR are amenable to laser therapy. Thus, management of a patient with CSR becomes highly individualized. Correct diagnosis of the condition and evaluation with multimodal imaging is crucial for providing proper patient care. The following cases explore diagnostic characteristics of patients with CSR using OCT imaging, fundus auto fluorescence, fluorescein angiography, and indocyanine green angiography. 


\section{OPTICAL COHERENCE TOMOGRAPHY}

The advent of OCT imaging has allowed clinicians to precisely detect CSR and monitor its clinical course by identifying characteristic features of the choroid and the retina. The most identifiable clinical finding in CSR is serous retinal detachment. On OCT imaging, this appears as an optically empty separation of the neurosensory retina from the RPE involving the macula (Figure 1). In addition, a pigmented epithelial detachment (PED) may present as a focal separation of the RPE from the underlying Bruch's membrane within the serous retinal detachment. This finding may be prominent or subtle and is regarded as the primary entry point for fluid to leak into the subretinal space (Figure 1). ${ }^{6}$

Figure 1: Two patients with CSR demonstrating serous retinal detachments (yellow arrows) with PEDs (red arrows). PEDs may be large and prominent as in patient $A$, or subtle and flattened in patient $B$.
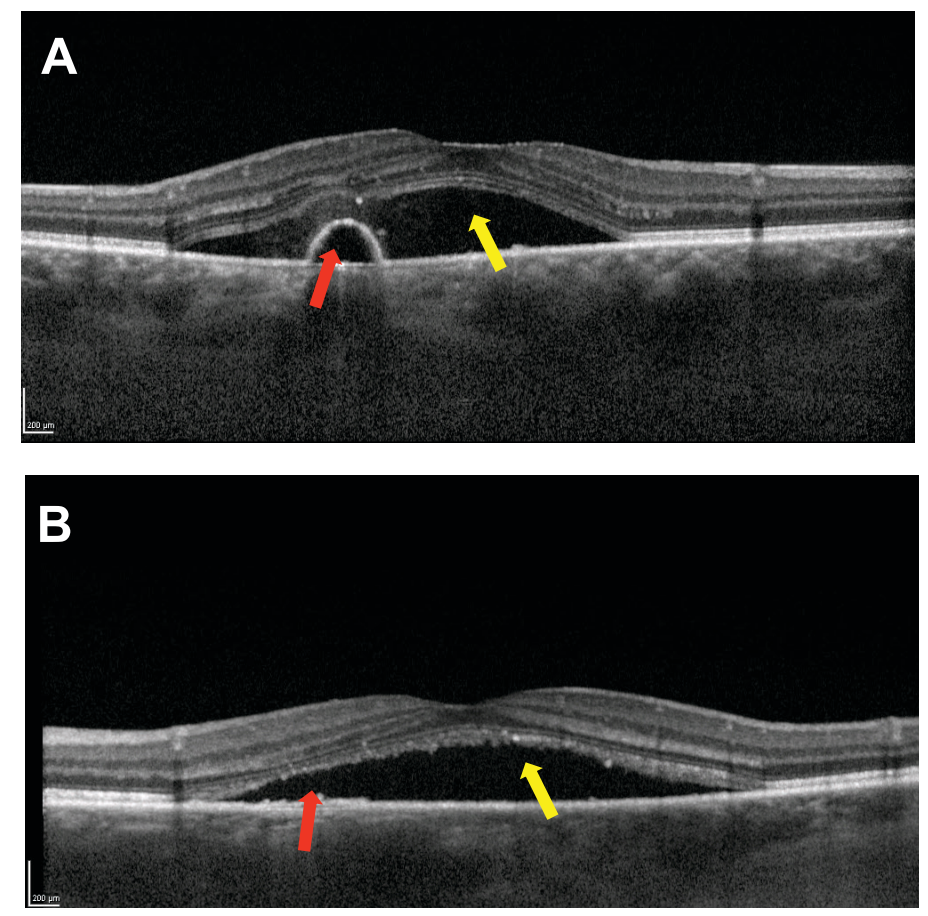

Enhanced depth imaging (EDI) allows for improved imaging of the choroid and repeatable measurement of choroidal thickness. This OCT imaging strategy is highly useful in the evaluation of patients with CSR, since increased choroidal thickness is a well-established finding in most patients with the condition (Figure 2). EDI has also led to the advent of the choroidal vascular index (CVI), which is the ratio of the luminal area, or vascular area of the choroid, to the total area of the choroid. Acute cases of CSR have been reported to have a higher mean CVI compared to age-matched norms and patients with chronic CSR. ${ }^{6}$ Patients with steroid-associated CSR also tended to have a higher average CVI than idiopathic cases. ${ }^{7}$ While CVI is currently used predominantly in research settings, it may in the future prove to be beneficial in understanding the pathophysiology of CSR in general practice.

Measurement of the choroidal thickness is indicated for a wide range of conditions, especially as it pertains to conditions in the pachychoroid spectrum. Conditions that are considered to be under this spectrum all share a characteristically thicker-than-average choroid and include pachychoroid pigment epitheliopathy, CSR, and polypoidal choroidal vasculopathy. ${ }^{8}$ Measurements are commonly taken directly underneath the fovea for repeatability. Normative values for the subfoveal choroidal thickness in the literature range from 272 to $448 \mu \mathrm{m}$, as thickness varies with refractive error, age, and ethnicity, though values above $400 \mu \mathrm{m}$ should arouse suspicion. ${ }^{9}$ For example, a 2018 study of 104 normal Iranian adults found a mean subfoveal choroidal thickness of $363 \pm 84 \mu \mathrm{m}$, while another 2008 study of American adults found an average subfoveal thickness of $287 \pm 76 \mu \mathrm{m} \cdot{ }^{10,11}$ Patients with active CSR demonstrate a greater subfoveal choroidal thickness compared to their resolved values. Chung et al. in South Korea found an average thickness of $446.8 \pm 101.0$ in active cases versus $414.7 \pm 93.9$ in resolved states. ${ }^{12}$ General trends to consider include thinning with age and thinning with increased axial length. 
Figure 2: OCT with enhanced depth imaging demonstrating above average choroidal thickness in patient with CSR

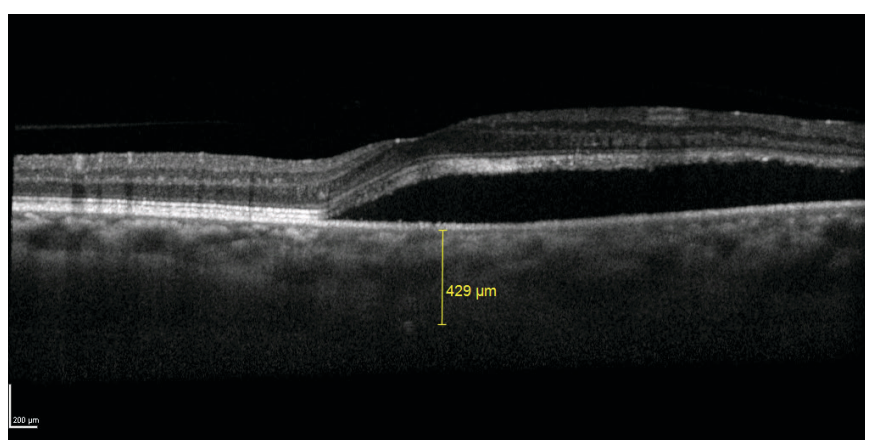

OCT imaging can also provide diagnostic clues to the chronicity of the disease. In the case of chronic serous detachment, photoreceptors remain detached from the RPE for an extended period, resulting in inadequate phagocytosis of their outer segments. Elongation of the outer segments leads to the OCT finding termed "shaggy photoreceptors" (Figure 3). ${ }^{13}$ It is important to note that shaggy photoreceptors are not unique to CSR and are also visible in other forms of chronic detachment, such as chronic serous detachments from choroidal melanoma. ${ }^{14}$

Figure 3: A) Acute serous detachment from CSR with minimal elongation of photoreceptor outer segments (yellow arrow) $B, C)$ chronic serous detachments with finding of "shaggy photoreceptors" (red arrows) from outer segment elongation
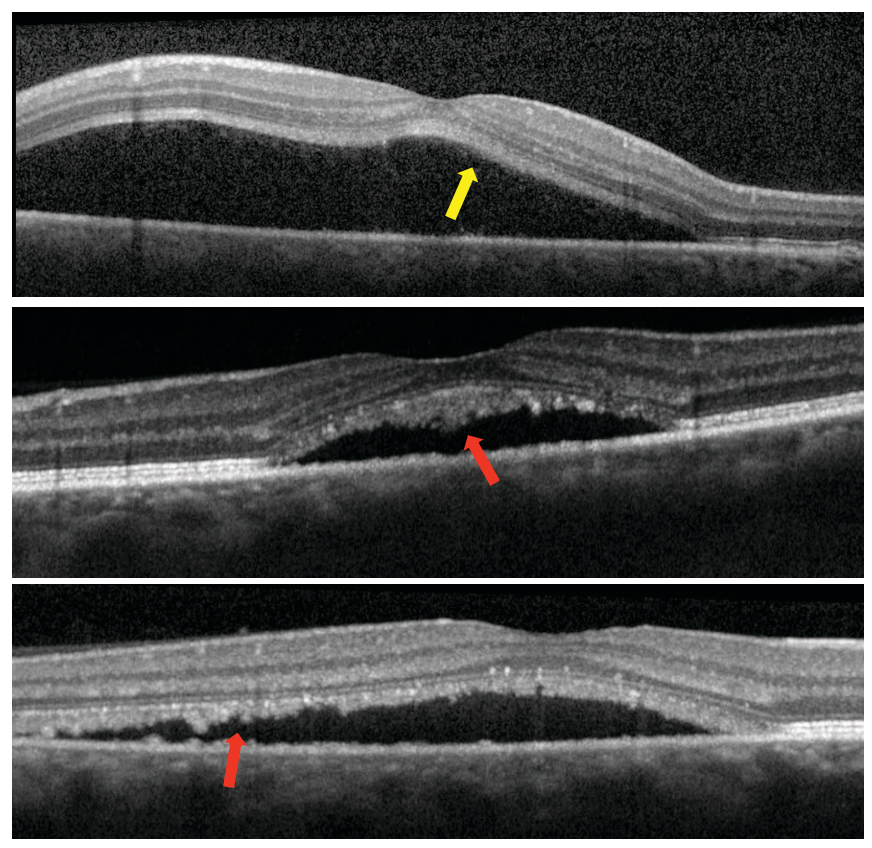

In addition to shaggy photoreceptors, patients with chronic disease may develop geographic atrophy, and cystic retinal edema notable on OCT imaging. These are clues of a very longstanding condition. In the case of significant central RPE and photoreceptor atrophy, more aggressive treatment may be unnecessary, since the visual prognosis remains poor regardless of intervention.

\section{OCT-ANGIOGRAPHY}

Patients with central serous retinopathy are also at risk of developing choroidal neovascular membranes (CNV), with estimates ranging between $2-9 \%$ of chronic cases..$^{15}$ Determining the origin of apparent fluid, either from the disease itself or from the development of CNV, can be difficult. The ability to perform detailed imaging with OCT is 
useful in these cases. OCT-angiography (OCT-A) imaging is a non-invasive method for detecting pathologic alterations in vasculature without dye injection. OCT-A provides vital structural information deep to the RPE that is typical of conventional OCT imaging, as well as a targeted analysis of abnormal blood flow characteristics, with a sensitivity similar to that of fluorescein angiography. ${ }^{15}$ The practitioner can target the area of suspected CNV by OCT and assess the underlying choriocapillaris for neovascularization. CNV is becoming increasingly well-characterized in OCT-A and may increase in utility due to its safety and relative ease of use in comparison to fluorescein angiography. OCT-A may also prove useful in cases without neovascularization. A recent study provided evidence demonstrating both hyper- and hypoperfusion at the level of the choriocapillaris in regions of serous detachment, as well as the regression of perfusion after the resolution of sub-retinal fluid. ${ }^{9}$ This suggests that OCT-A can be used to assess the risk of CSR progression by monitoring the pattern of flow abnormalities.

\section{FUNDUS AUTOFLUORESCENCE}

Fundus autofluoresence imaging is a useful tool in the evaluation of CSR. This technique collects light-emission data using blue-light excitation to visualize fluorophore content (i.e., melanin or lipofuscin) throughout the retina. FAF is used to identify drusen and abnormalities of the outer retina and RPE which would otherwise be difficult to detect under funduscopic examination, fundus photography, or fluorescein angiography. ${ }^{16,17}$ Abnormalities are differentiated from one another by their signal intensity as hyper-autofluorescent, hypo-autofluorescent, and iso-autofluorescent.

In acute cases of CSR, accumulated subretinal fluid may create a blocking effect resulting in a region of hypo-autofluorescence (Figure 4). The extent of serous detachment may be more readily visualized on FAF than on clinical evaluation. In chronic cases, pinpoint areas of hyper-autofluorescence develop. These are thought to be precipitates of fluorophores from non-phagocytosed, elongated photoreceptor outer segments (Figure 4). As the condition becomes very longstanding or recurrent, damage to the RPE may present as both regions of hyper-autofluorescence correlating to unviable tissue and accumulated fluorophores and regions of hypo-autofluorescence corresponding to regions where RPE atrophy has already occurred. ${ }^{17}$ In chronic disease, the pattern of RPE disruption on FAF may take on an unusual appearance as serous fluid is shifted downward by gravity, resulting in hyper-autofluorescent "comet tails". ${ }^{13}$

Figure 4: A) Patient with acute CSR and well circumscribed region of fairly uniform hypo-autofluorescence corresponding to area of serous detachment B) Patient with chronic CSR and shaggy photoreceptors on OCT, showing resultant pinpoint areas of hyper-autofluorescence

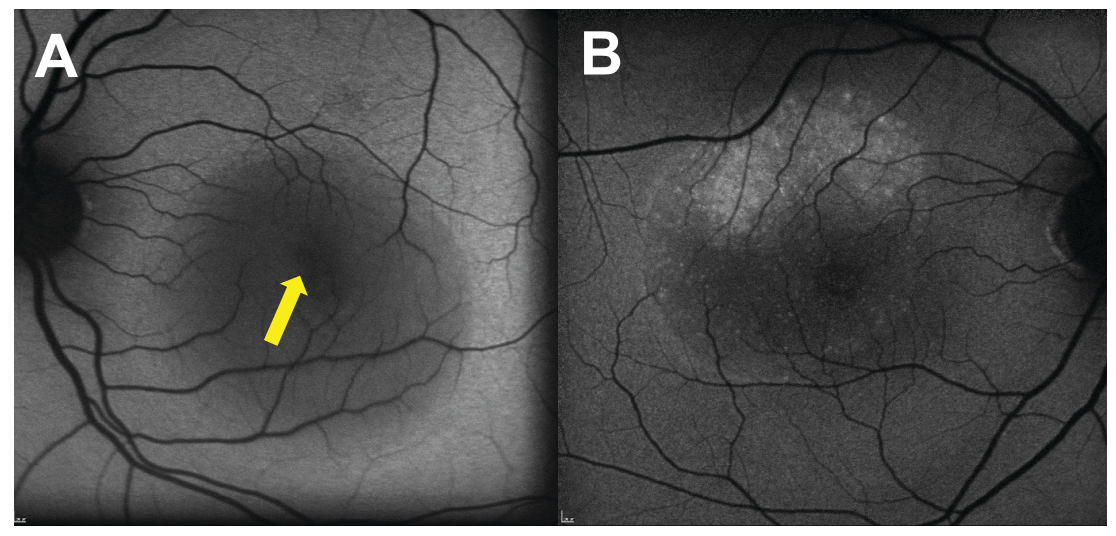

\section{FLUORESCEIN ANGIOGRAPHY}

Fluorescein angiography is useful for identifying areas of leakage, especially in cases with multiple detachments outside of the macula. Leakage can occur in an inkblot pattern which begins as a focal pinpoint and enlarges in the late phase (Figure 5). Leakage can also manifest as a smoke-stack appearance similar to cystoid macular edema, which also begins as a focal point but grows in a cloud-like form into the late phase (Figure 6). The smoke-stack form is not as common in CSR and appears in 10-15\% of acute cases. ${ }^{2}$

The identification of PEDs by fluorescein angiography can be vital, especially when considering focal laser coagulation. If the area of leakage is a sufficient distance from the fovea to be considered safe, laser application can shorten the duration by 2 months and can reduce the rate of recurrence. ${ }^{18,19}$ Navigated laser photocoagulation, such as with the Navilas" system (OD-OS GmbH, Teltow, Germany) provides eye-tracking and pre-rendered treatment-planning 
by working in conjunction with fundus photography and fluorescein angiography, and has been shown to be more accurate than conventional laser. ${ }^{19}$ Early laser use may not be indicated in all cases, however, and should be preceded by an observation period past the 3 to 4 month period. ${ }^{20}$

Figure 5: A) Early pinpoint area of leakage @ 30 seconds B) Increased hyper fluorescence throughout later stages in ink blot pattern C) Area of leakage corresponds to PED seen on OCT.
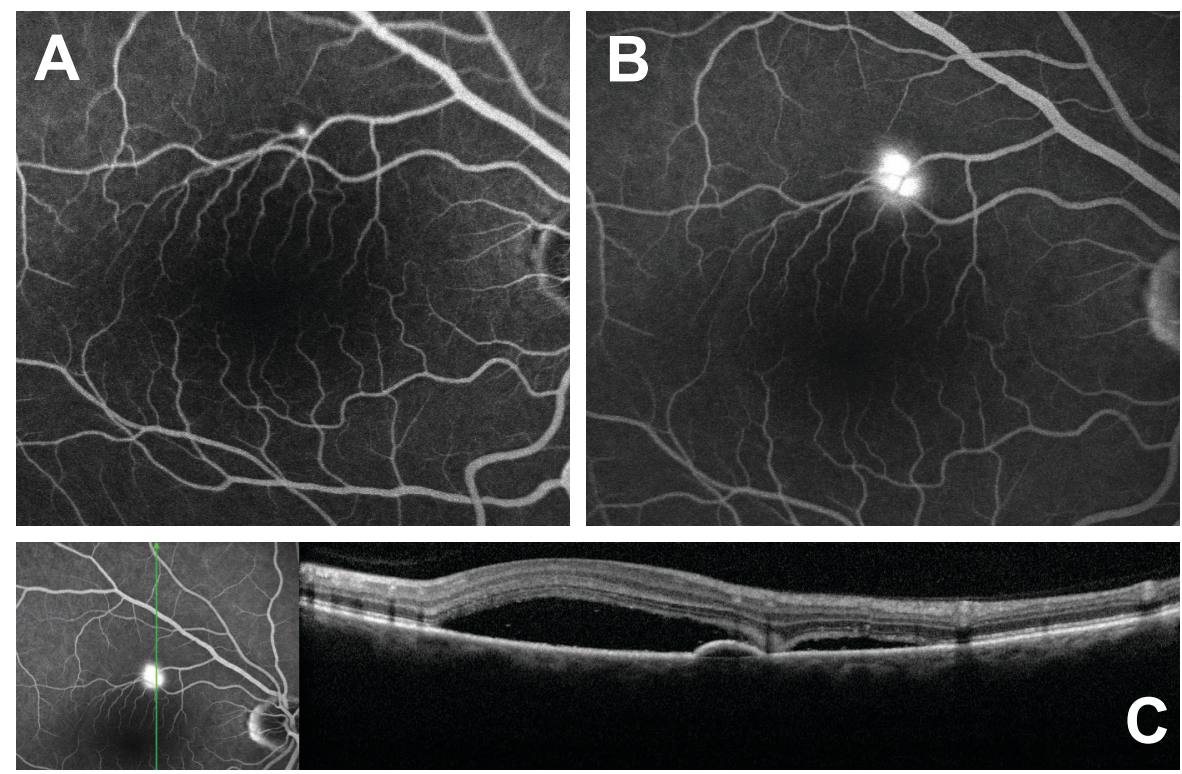

Figure 6: Smoke stack pattern of leakage
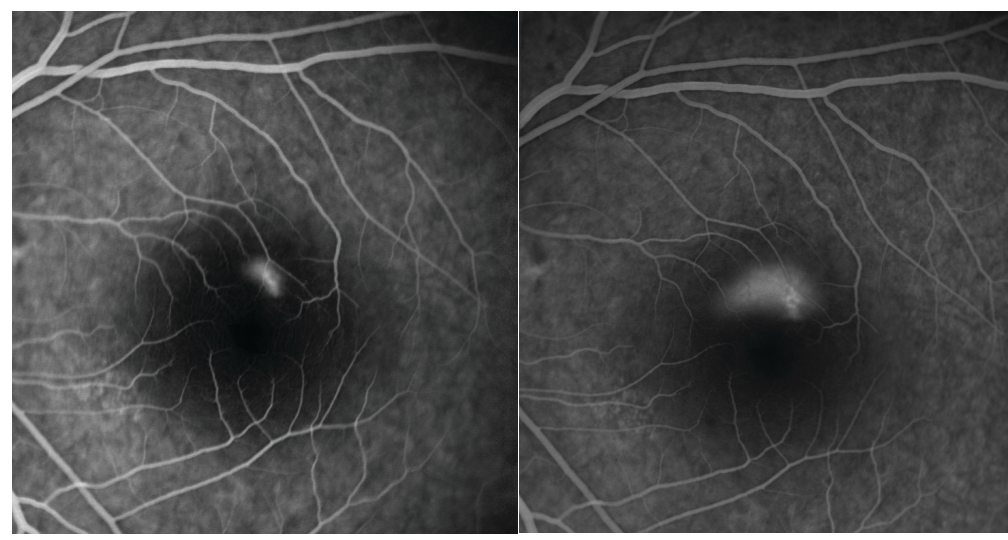

\section{INDOCYANINE GREEN ANGIOGRAPHY}

Indocyanine green (ICG) angiography is another intravenous imaging method that can be performed concurrently with fluorescein angiography and highlights choroidal vascular changes. ICG exhibits close to $98 \%$ plasma binding, resulting in substantially greater retention of the dye compared to fluorescein in the otherwise leaky choroidal vasculature. ${ }^{2}$ The use of ICG is particularly useful in combination with photodynamic therapy (PDT), since the increased visualization of the choroidal vasculature allows for more accurate targeting of leakage. In CSR, there is delayed choroidal filling in the early phase and patches of non-perfusion, leading to choroidal venous dilation and geographic hyper-fluorescence in the mid phase (Figures 7, 8). ${ }^{21}$ These areas of hyper-permeable choroid correlate with areas of RPE leakage, but may also appear elsewhere in the retina. ${ }^{2}$ This abnormality in vascular filling is often seen bilaterally and more extensively across the posterior pole, in comparison to the focal changes seen in fluorescein imaging. ICG should be performed to rule out polypoidal choroidal vasculopathy (PCV) and other causes of neovascularization. 
PCV, which is characterized by abnormal choroidal vessels culminating in focal projections through the posterior retina in the region surrounding the optic nerve and/or macula, may present very similarly to CSR under FA, but is better characterized by ICG as having more confluent polyp-like lesions in the mid to late phase. ${ }^{2}$

Figure 7: A) ICGA at 30 seconds showing hypofluoresence centrally with dilated choroidal vessels. B) ICGA late stage at 14 minutes shows localized area of hyperfluorescence with potential to target region with focal laser.

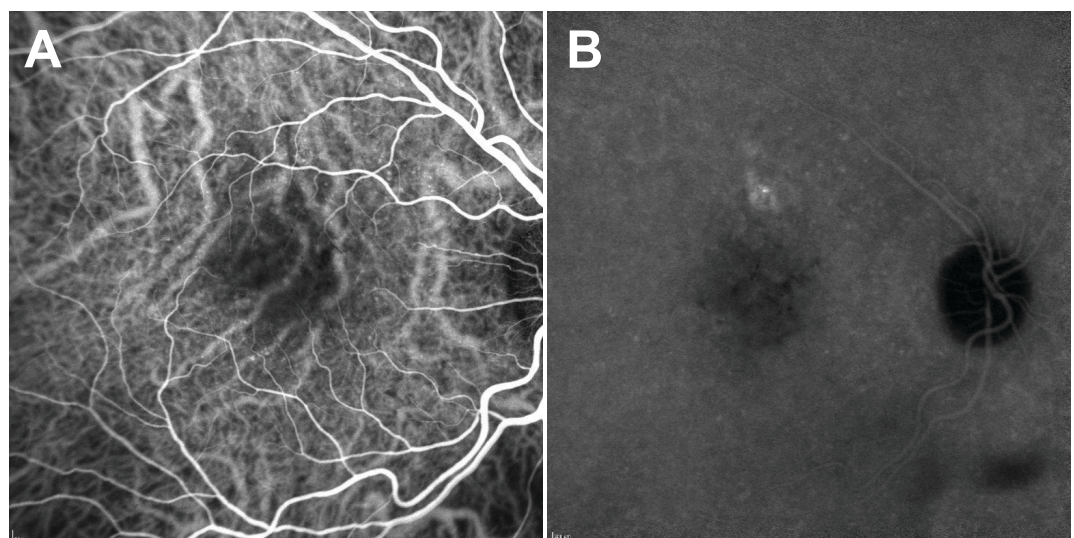

Figure 8: A) Early ICGA showing regions of hypofluorescence and dilated choroidal vessels. B) Late stage ICGA showing regions of leakage. Larger regions or regions near fovea cannot be targeted with focal laser, but have potential for treatment with PDT.

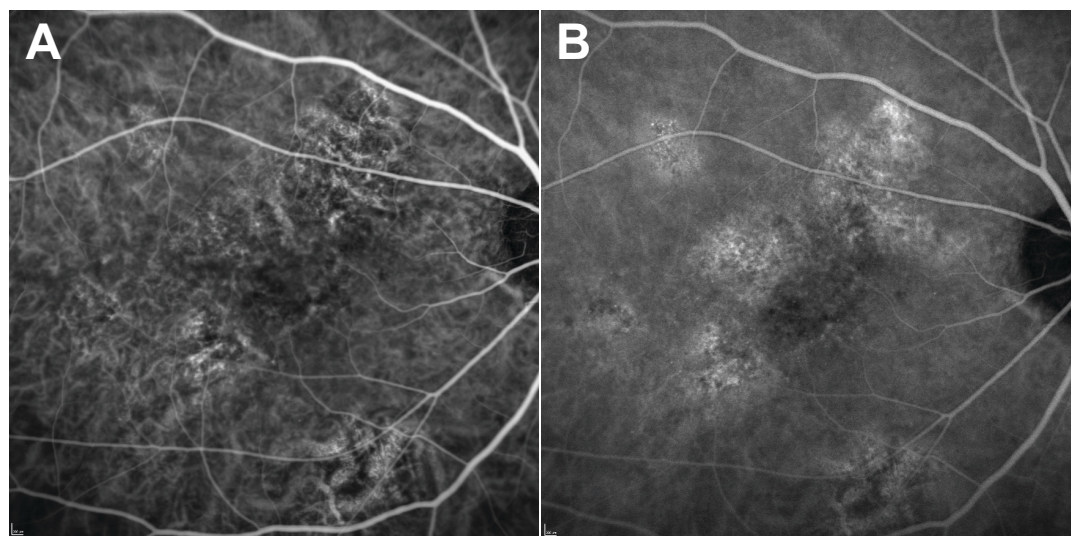

CASE 1

A 65-year-old African-American male presented with complaints of very poor vision OD and mild blur OS with his habitual spectacles. He reported that the right eye had had poor vision for 15 years, and he had been previously told by several eye doctors that his vision would not improve with treatment. He denied any history of trauma and vaguely recalled a diagnosis of "macular degeneration" in the right eye causing his vision loss. He denied any history of treatment.

He had been undergoing chemotherapy and radiation for an unspecified lesion in the brain and for lung cancer for the preceding year and felt his vision had decreased over that period. His medical history was additionally remarkable for Type 2 Diabetes diagnosed 3 years prior with a recent HbAlc of $6.3 \%$, hypertension, and history of a minor cerebrovascular accident 5 years prior. The patient denied a history of ocular disease in the family and denied tobacco, alcohol, or recreational drug use. The patient's medications included $10 \mathrm{mg}$ cetirizine prn, $50 \mathrm{mg}$ sildenafil prn, $2.5 \mathrm{mg}$ apixaban bid, $1000 \mathrm{IU}$ cholecalciferol qd, $10 \mathrm{mg}$ hydrocodone/ $325 \mathrm{mg}$ acetaminophen (Norco) qd, 500 $\mathrm{mg}$ levetiracetam bid, $50 \mathrm{mg}$ losartan bid, $50 \mathrm{mg}$ metoprolol qd, $5 \mathrm{mg}$ tradjenta qd, $20 \mathrm{mg}$ omeprazole qam, $0.4 \mathrm{mg}$ tamsulosin qhs, and $1 \mathrm{mg}$ dexamethasone bid. He was allergic to morphine.

He had been seen in our clinic 16 years prior, and records indicated a diagnosis of unspecified macular degeneration with hard drusen and suspicion of macular edema. His primary complaint at that visit was acute blur OU upon 
waking beginning 5 days prior. Best corrected visual acuity was 20/70- OD and 20/25+ OS. He was scheduled for follow-up with fluorescein angiography, but did not return.

At the current visit, vision was count-fingers at 3 feet OD and 20/30-2 OS with no improvement in visual acuity with pinhole or refraction. Extraocular muscles demonstrated full range of motion in both eyes without pain or diplopia.

Intraocular pressure was $16 \mathrm{mmHg} \mathrm{OD}$, OS. Anterior segment examination revealed grade 2 nuclear sclerotic cataracts in each eye, but was otherwise unremarkable. There was no neovascularization of the iris in either eye. Dilated posterior segment evaluation revealed healthy, symmetric optic nerves without pallor with a C/D ratio graded 0.40 rd OD and $0.45 \mathrm{rd} \mathrm{OS}$. The vitreous was clear and the peripheral retina was intact. Vasculature demonstrated tortuosity of the arteries and veins OU. There was no evidence of diabetic retinopathy OU.

The right eye was remarkable for a "window" RPE defect in the fovea, approximately $3 / 4$ of a disc diameter in size. The lesion was well-demarcated and encircled by a faint ring extending downwards to the inferior arcades with pinpoint yellow precipitates resembling exudates. In the left eye, the superior arcade and macula had drusen-like deposits (Figure 9).

Figure 9: Fundus photography and corresponding fundus autofluorescent imaging:

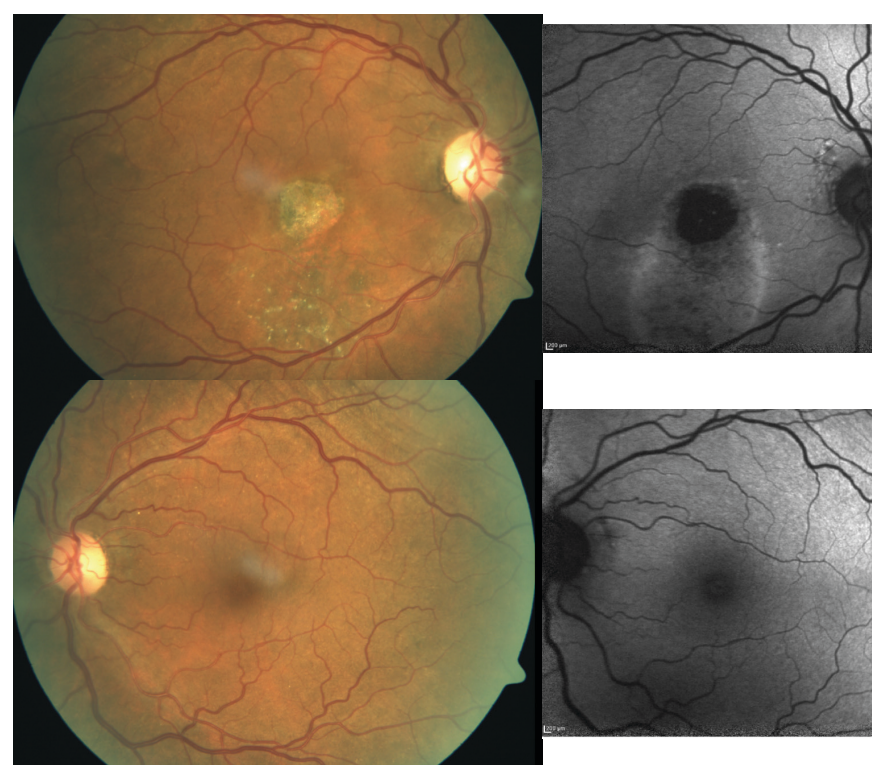

Fundus autofluoresence (FAF) OD demonstrated dense, homogenous hypo-autofluoresence of the foveal lesion consistent with complete atrophy of the RPE. In addition to this area, RPE damage extended inferiorly in a clearly defined hyper-autofluorescent pattern indicating a gravitational tract towards the inferior arcade. There were also hypo-autofluorescent spots representative of the pseudo-exudative changes within the ring. FAF OS demonstrated even iso-autofluorescent macular pigmentation (Figure 9).

SD-Optical coherence tomography (OCT) imaging of the macula OD demonstrated cystic alterations in the fovea altering both the inner and outer retinal anatomy with significant outer retina and photoreceptor atrophy underneath. A small, shallow detachment of the RPE (PED) was evident inferior to the fovea as well as pronounced outer retinal loss extending as far anteriorly as the inner nuclear layer and through the RPE. OCT of the macula OS was unremarkable for edema and retinal layers remained intact with preservation of the foveal contour.

The patient's findings were consistent with chronic central serous retinopathy OD. He was educated on the condition and the continued poor visual prognosis despite treatment. He was counseled regarding avoidance of CSR triggers such as use of steroids including nasal sprays. However, we recommended that the patient continue use of dexamethasone as it was adjunct to his chemotherapy. The patient desired consultation for treatment options, however, and was referred to a retinal subspecialist for evaluation. The patient refused dilation at his follow-up with the retinologist and did not return for follow-up. 
Figure 10: A. Cystic edema overlying region of photoreceptor and RPE atrophy in the fovea.; B.Shallow PED (yellow arrow); C. Outer retinal atrophy extending inferiorly; $D$. Normal macular OCT in OS
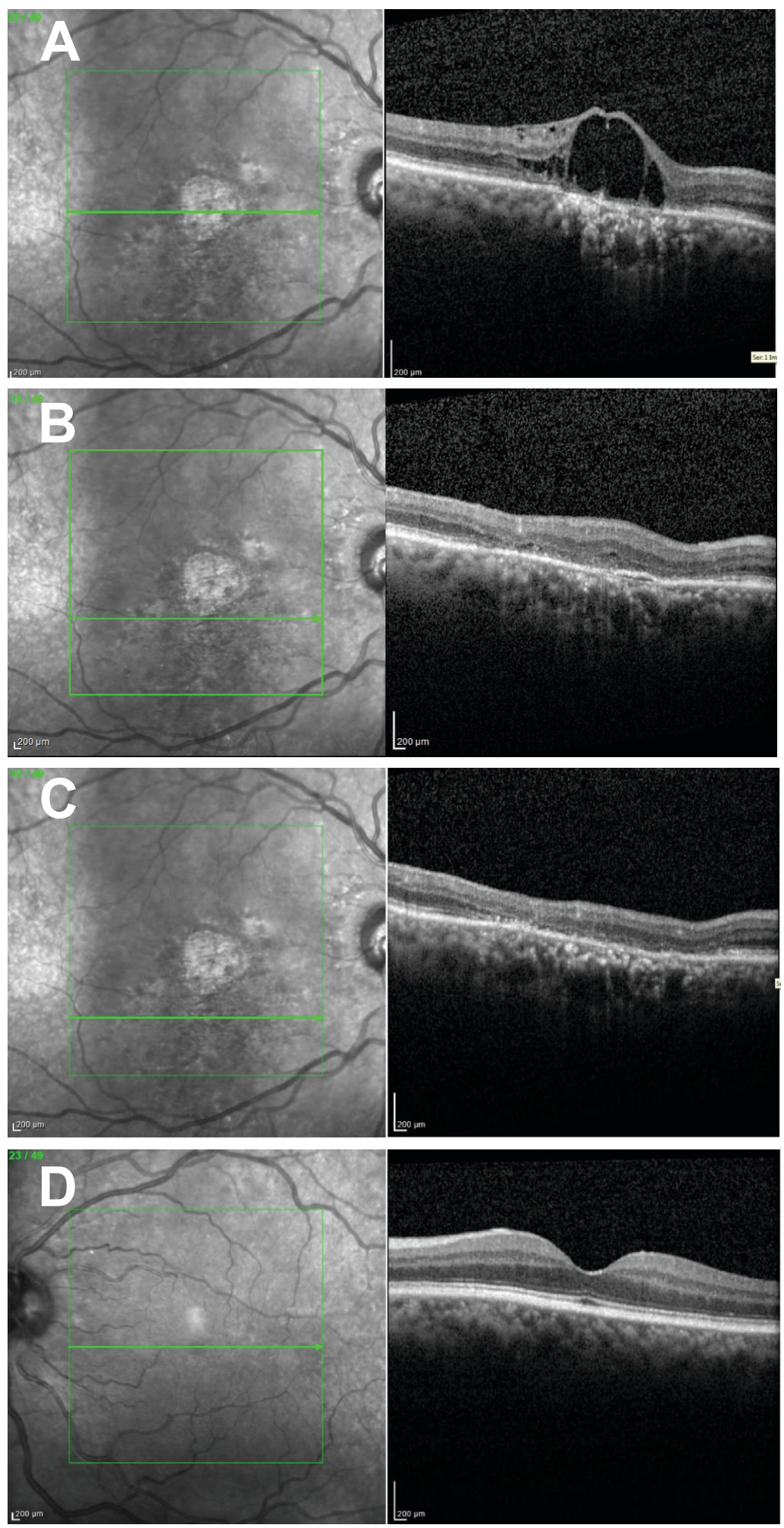
CASE 2

A 38-year-old African-American male presented with a brown spot in his central vision OD, which had begun 1 week prior. He described his vision as if he was looking through a coffee stain. The spot did not move and followed his fixation. The patient denied symptoms in his left eye. He denied a history of ocular disease, injury, or abnormal conditions for himself and his immediate family. His medical history was remarkable for hypertension and ADHD. His current medications included $5 \mathrm{mg}$ amphetamine/ $5 \mathrm{mg}$ dextroamphetamine qd, $40 \mathrm{mg}$ atomoxetine qd, and $500 \mathrm{mg}$ naproxen bid. Pupils, EOMs, and confrontation visual fields were unremarkable OD, OS. Best corrected visual acuity was 20/20- OD and 20/20 OS. Amsler grid testing was remarkable for a brown spot centrally and nasal to fixation.

Intraocular pressure was $18 \mathrm{mmHg}$ OD, $20 \mathrm{mmHg}$ OS. Anterior segment examination was unremarkable OD, OS. Dilated examination of the posterior segment was remarkable for a large well-circumscribed non-pigmented ring around the fovea OD, which was greater inferiorly (Figure 10). The macula OS was unremarkable. The C/D ratio of the optic nerves was $0.30 \mathrm{rd}$ OD and OS. The periphery was unremarkable for holes, breaks, or tears and the vasculature was of normal caliber OU.

OCT imaging of the macula OD revealed a neurosensory detachment 8 to 10 disc diameters in size extending from the temporal macula to just inferior to the fovea (Figures 11,12). OCT demonstrated subfoveal choroidal thickness greater than 450 microns (outside of the accepted normative range) and a small PED present within the serous detachment. ${ }^{27}$ There was minimal elongation of the photoreceptor outer segments.

The patient was diagnosed with central serous chorioretinopathy OD. Both the patient history and OCT findings suggested an acute episode. He admitted to recent stress at home and work, possibly precipitating his symptoms, and denied any recent steroid use. It was decided to observe the patient with serial OCT imaging.

Over the next two visits and a span of two months, the patient's visual symptoms persisted without improvement. Visual acuity remained 20/20- OD, OS. Serial comparison of OCT imaging demonstrated migration of the fluid inferiorly, a common finding in CSR, but persistent central subretinal fluid remained. Due to the patient's symptoms and persistence of central subretinal fluid, the patient was started on oral eplerenone $50 \mathrm{mg}$ bid for 4 weeks at his second visit after verifying that he was not taking anti-hypertensive medication at the time. Treatment did not encourage fluid resorption based on serial OCT imaging nor did it reduce the patient's symptoms.

The patient was scheduled in our optometric fluorescein angiography clinic. The angiography study demonstrated an area of early focal pooling along the superior temporal arcade OD that increased in fluorescence through the mid and late phases in an ink blot pattern (Figure 13). The area of pooling correlated with the small PED on OCT and confirmed a break in the Bruch's-RPE complex. Due to the persistence of the condition despite observation and eplerenone, along with patient frustration and subjective visual disturbance, the patient was referred to ophthalmology for treatment options. The patient did not present for follow-up.

Figure 11: Fundus findings $O D$.

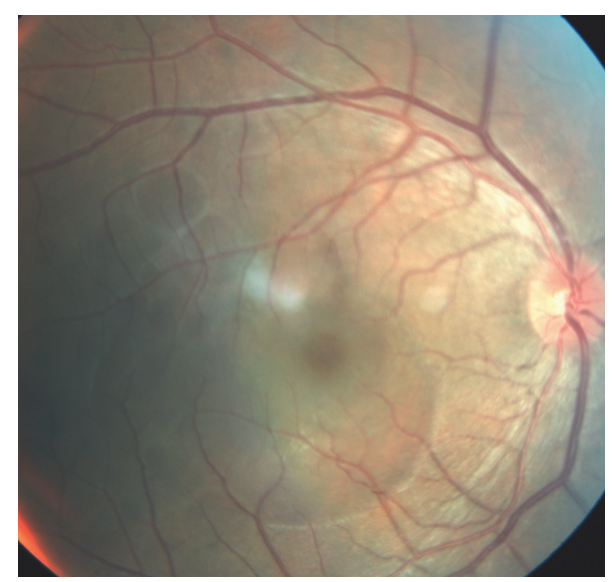


Figure 12: Comparing progression of horizontal slices with macula OCT. A) Persistent subretinal fluid over a period of 3 months of a region inferior to the macula.
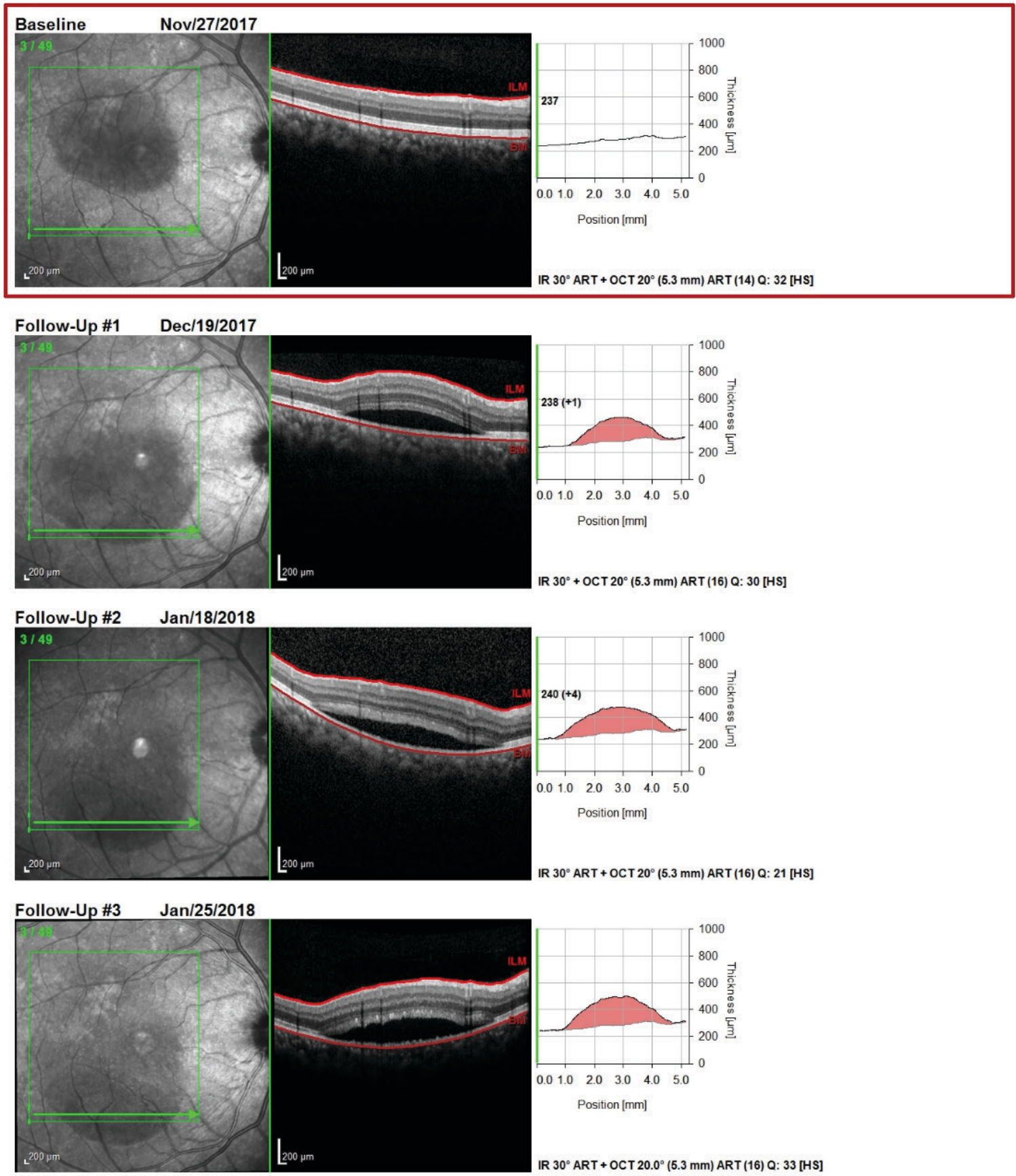
Figure 12:B) Horizontal section through the fovea demonstrating persistent subretinal fluid causing progressive serous detachment and expansion of the affected retina coinciding with net decrease in macular thickness.
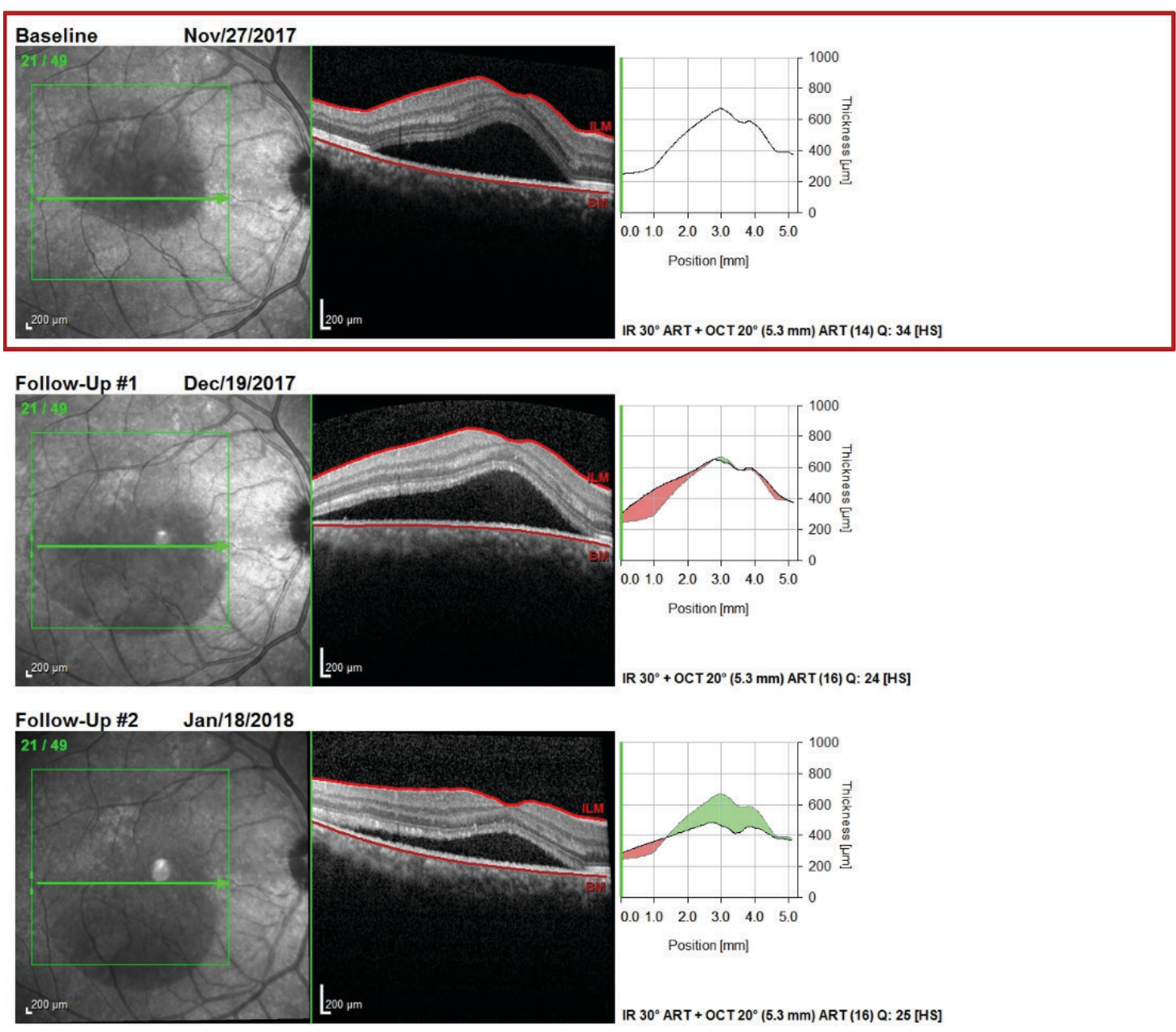

Follow-Up\#3 Jan/25/2018

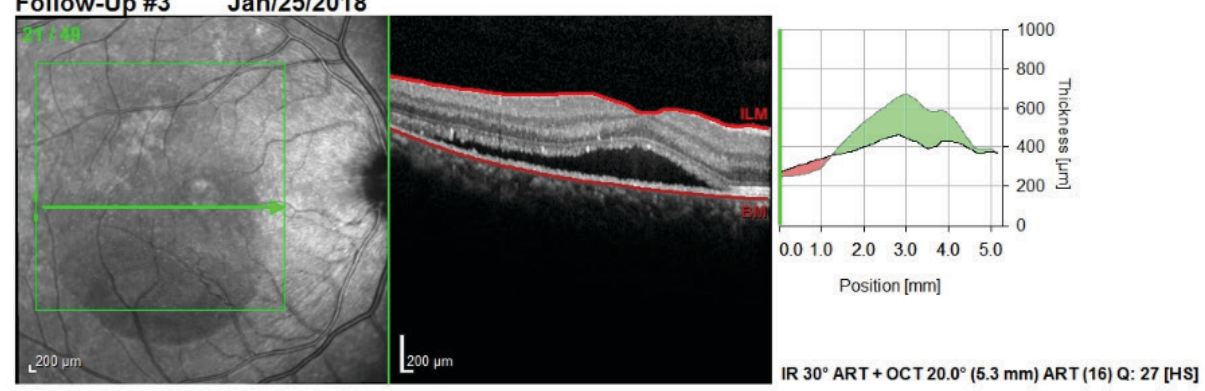


Figure 13: Left to right, top to bottom shows increased fluorescence from early stages to late stages in ink blot pattern.
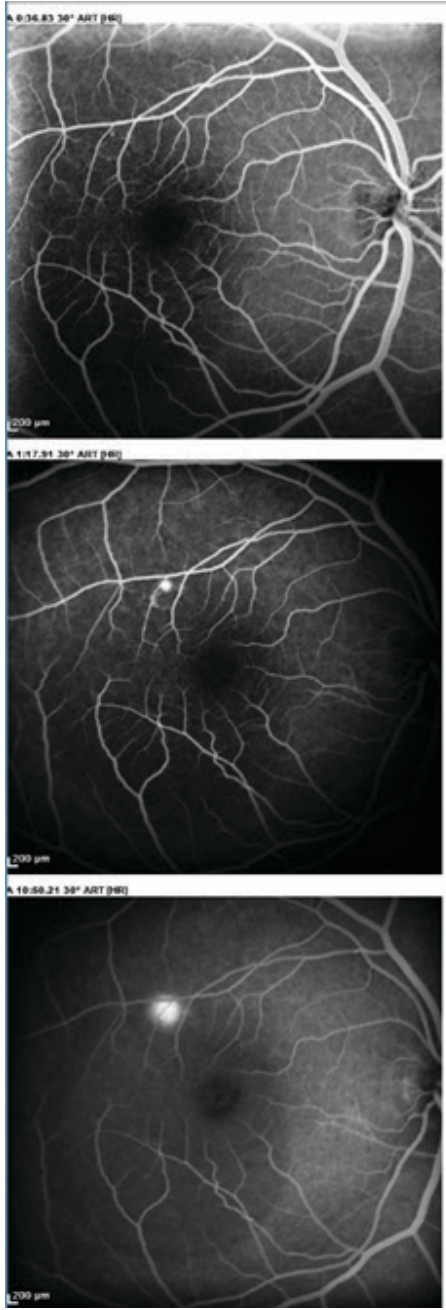
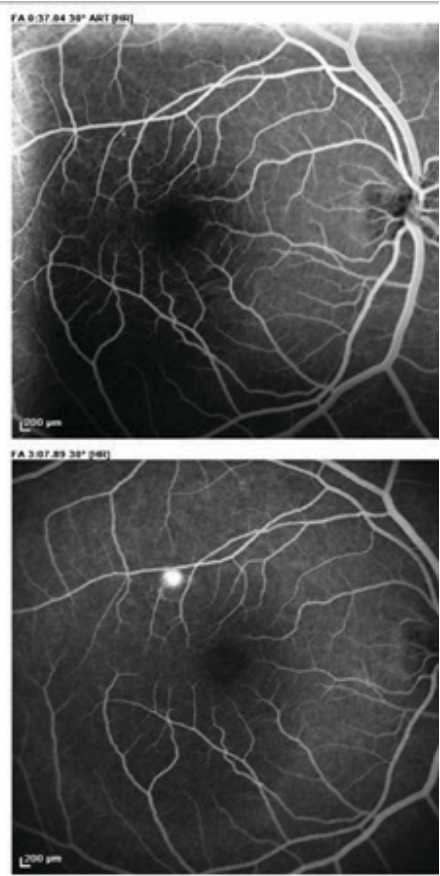

\section{DISCUSSION}

The pathophysiology of CSR can be generally described as an abnormality in choroidal circulation resulting in a disruption of the RPE and neurosensory retina interaction. In the event of stasis, choriocapillaris nonperfusion, or inflammation, an alteration in hydrostatic pressure may cause disruption of the RPE, allowing fluid to enter the subretinal space and causing a neurosensory detachment. Focal detachments of the RPE (PED), which can present in isolation or scattered throughout the posterior pole, are often targets of treatment in focal laser coagulation to reduce the influx of subretinal fluid..$^{21,22}$ Increased choroidal thickness has been proposed to be the result of an increase in choroidal permeability, causing a hyperpermeable state that is more susceptible to leakage. ${ }^{5}$

One currently accepted hypothesis regarding the role of corticosteroids in CSR states that the alteration of glucocorticoid and mineralocorticoid receptor activity in the choroid and the RPE can lead to water and sodium retention, which is responsible for serous neurosensory detachment. ${ }^{23}$ Aldosterone, the primary mineralocorticoid in the body, has been shown to induce oxidative stress, inflammation, and endothelial dysfunction in low doses and may affect expression in retinal glial Muller cells in ion and water exchange. ${ }^{23}$ Intravitreal injection of glucocorticoids has not been associated with a higher incidence of CSR, suggesting a higher-order metabolic etiology, though there have been reported cases of aggravation of pre-existing CSR with intravitreal triamcinolone..$^{23,24}$

In acute CSR, which is commonly defined as resolving within 3 to 4 months of presentation, sustained visual symptoms are often limited, since foveal integrity is preserved even in the event of foveal involvement. ${ }^{25}$ Initial management for acute cases, such as with patient \#1, is often observed. In a 2017 study, Daruich et al. evaluated 31 treatment-naïve patients 
at their initial episode, of whom $84 \%$ had resolution without intervention by 6 months. ${ }^{26}$ After resolution, however, up to $50 \%$ of patients develop recurrence in the same eye within a year of initial presentation and are more likely to recur if they have a history of depression and/or psychiatric illness. ${ }^{4}$ Patients with a history of resolved acute CSR may still present with visual sequelae such as metamorphopsia and reduced contrast and color vision due to the structural alteration of the photoreceptor layer and reduced cone density. ${ }^{5}$ This damage worsens in chronic and recurrent cases.

If there is persistent subretinal fluid lasting longer than 3 to 4 months, the condition is regarded as chronic and often necessitates intervention. Persistent separation of the neurosensory retina leads to atrophy of the outer segments in the photoreceptor layer, causing permanent outer retinal damage. ${ }^{7}$ Hyper-reflective precipitations, such as those seen in Figure 9, result from this outer segment atrophy and are characteristic of chronic disease. They have been theorized to result from the accumulation of proteins and macrophages from the phagocytosed outer segments that migrate inferiorly under the influence of gravity. ${ }^{14}$ Cystoid, bullous macular edema, as seen in patient \#1, has been associated with disease duration longer than 5 years and is a negative prognostic indicator. ${ }^{27}$ Outer retinal layer loss and granular deposits anterior to the RPE are also associated with chronic edema. Chronic disease may lead to RPE atrophy, presenting as marked regions of hypo-autofluorescence on FAF, also seen in patient \#1. The formation of choroidal neovascular membranes can also complicate the course of disease. In patients with such extensive outer retinal disease, there is a poor prognosis despite treatment. Treatment is aimed at hastening visual recovery in addition to preventing long-term negative sequelae.

Oral medications have been targeted as a non-invasive method to shorten the course of the disease, with varying results. Among aldosterone inhibitors, spironolactone and eplerenone have been considered. These are steroidal anti-mineralocorticoids that are used to treat chronic heart failure and hypertension. They act as diuretics by blocking the binding of aldosterone to the mineralocorticoid receptor via competitive inhibition and may have some application in acute cases. ${ }^{21}$ Zucchiatti et al. in 2018 claimed higher and faster resolution with eplerenone compared to observation in cases of acute CSC, albeit in a small sample size ( $\mathrm{n}=27$ eyes). ${ }^{28}$ While these findings have not yet been substantiated in other literature, this may be a beneficial first-line therapy in patients without timely resolution. Further randomized, multicenter prospective trials would be beneficial in guiding this treatment.

Additional oral medications have been considered, including aspirin, ketoconazole, mifepristone, finasteride, rifampin, and acetazolamide. ${ }^{29,30}$ While there is limited evidence to support any of these therapies, a non-randomized, open label study by Caccavale et al. in Italy demonstrated that a $100 \mathrm{mg}$ daily dose of aspirin for 1 month followed by $100 \mathrm{mg}$ every other day for 5 months resulted in faster visual recovery and fewer recurrences compared to the control group. ${ }^{30}$ Because increased hypercoagulability and platelet aggregation have been associated with an increased risk of CSR, the authors suggested a 6-month course of low-dose aspirin to decrease the time of resolution due to its anti-aggregate properties.

The use of intravitreal anti-VEGF agents has been considered for the treatment of CSR. While there is clear benefit to the use of anti-VEGF in patients who develop CNV secondary to CSR, the use of anti-VEGF for treatment of the disease itself remains controversial. The fact that there is no increase in the level of VEGF in the vitreous of patients with CSR remains a point of contention for those opposed to the use of anti-VEGF for CSR. ${ }^{31} \mathrm{~A}$ meta-analysis by Chung et al., which included four studies comparing intravitreal bevacizumab to monitoring, did not conclusively identify a benefit of anti-VEGF in acute or chronic CSR. ${ }^{31}$

The use of PDT has been widely accepted as an effective method of promoting the absorption of subretinal fluid in CSR by targeting abnormalities in choroidal vasculature via intravenous injection of the photosensitive drug verteporfin and use of a laser that selectively targets verteporfin. This minimizes collateral damage, and can be applied to broader areas and more central areas than focal laser. In a 2018 study, an anatomic response was evident in $96 \%$ $(n=39)$ of cases of chronic CSR with preservation of the outer nuclear layer and ellipsoid zone. ${ }^{22}$ The application of PDT is guided by either fluorescein angiography or indocyanine green angiography to target leaking areas.

Focal thermal laser targeting local areas of leakage and PEDs has been shown to be beneficial in CSR. Laser treatment is often guided by fluorescein or indocyanine green angiography. While there is evidence of a decreased time for the reabsorption of subretinal fluid, it is uncertain whether there is a reduced rate of recurrence. A focal laser cannot be applied to broad regions or regions that are too close to the fovea. ${ }^{22,29}$

\section{CONCLUSION}

While most cases of acute CSR are self-limiting and require no intervention, chronic and recurrent cases can result in permanent vision loss. When appropriately applied, medical intervention may help decrease the duration of the condition and the rate of recurrence, ultimately improving visual outcomes. The use of multimodal imaging allows for a more accurate diagnosis of the condition by ruling out other causes of serous retinal detachment. In addition, 
it allows for a determination of the chronicity of the condition and improves the ability to tailor treatment for each individual case. FAF, OCT, fluorescein angiography and ICG angiography each reveal a unique piece of the clinical picture and provide a physiological perspective that is invaluable in determining both the patient's prognosis in CSR and the best targeted therapy. The practitioner should be mindful of the various treatment options available and refer appropriately, especially in chronic cases, to avoid sight-threatening complications.

\section{ACKNOWLEDGEMENT}

The authors would like to thank Andrew Rixon, OD, FAAO for his support and advice during the preparation of this manuscript.

FINANCIAL DISCLOSURES

The authors have no financial interests and declare no conflicts of interest in this work.

\section{REFERENCES}

1. Liegl R, Ulbig MW. Central serous chorioretinopathy. Ophthalmologica 2014;232(2):65-76.

2. Schachat AP, ed. Ryan's Retina. Sixth edition. Edinburgh ; New York: Elsevier; 2018.

3. Wang M, Munch IC, Hasler PW, Prünte C, Larsen M. Central serous chorioretinopathy. Acta Ophthalmol 2008;86(2):126-145.

4. Liew G, Quin G, Gillies M, Fraser-Bell S. Central serous chorioretinopathy: a review of epidemiology and pathophysiology: Central serous chorioretinopathy. Clin Exp Ophthalmol 2013;41(2):201-214.

5. Iacono P, Battaglia Parodi M, Falcomatà B, Bandello F. Central serous chorioretinopathy treatments: a mini review. Ophthalmic Res 2015;55(2):76-83.

6. Rasheed M, Goud A, Mohamed A, Vupparaboina K, Chhablani J. Change in choroidal vascularity in acute central serous chorioretinopathy. Indian J Ophthalmol 2018;66(4):530.

7. Ambiya V, Goud A, Rasheed MA, Gangakhedkar S, Vupparaboina KK, Chhablani J. Retinal and choroidal changes in steroid-associated central serous chorioretinopathy. Int J Retina Vitreous 2018;4(1).

8. Ersoz MG, Karacorlu M, Arf S, Hocaoglu M, Sayman Muslubas I. Pachychoroid pigment epitheliopathy in fellow eyes of patients with unilateral central serous chorioretinopathy. Br J Ophthalmol 2018 Apr;102(4):473-478.

9. Seo EJ, Um T, Yoon YH. Abnormal choroidal flow on optical coherence tomography angiography in central serous chorioretinopathy. Clin Exp Ophthalmol 2018 Dec 5. doi: 10.1111/ceo.13454. [Epub ahead of print].

10. Entezari M, Karimi S, Ramezani A, Nikkhah H, Fekri Y, Kheiri B. Choroidal thickness in healthy subjects. J Ophthalmic Vis Res 2018;13(1):39.

11. Margolis R, Spaide RF. A pilot study of enhanced depth imaging optical coherence tomography of the choroid in normal eyes. Am J Ophthalmol 2009;147(5):811-815.

12. Chung Y-R, Kim JW, Choi S-Y, Park SW, Kim JH, Lee K. Subfoveal choroidal thickness and vascular diameter in active and resolved central serous chorioretinopathy. Retina 2018;38(1):102-107.

13. Matsumoto H, Kishi S, Otani T, Sato T. Elongation of photoreceptor outer segment in central serous chorioretinopathy. Am J Ophthalmol 2008;145(1):162-168.el.

14. Matsumoto H, Kishi S, Sato T, Mukai R. Fundus autofluorescence of elongated photoreceptor outer segments in central serous chorioretinopathy. Am J Ophthalmol 2011;151(4):617-623.el.

15. Bonini Filho MA, de Carlo TE, Ferrara D, et al. Association of choroidal neovascularization and central serous chorioretinopathy with optical coherence tomography angiography. JAMA Ophthalmol 2015;133(8):899.

16. Spaide R, Klancnikjr J. Fundus autofluorescence and central serous chorioretinopathy. Ophthalmology 2005;112(5):825-833.

17. Yung M, Klufas MA, Sarraf D. Clinical applications of fundus autofluorescence in retinal disease. Int J Retina Vitreous. 2016;2(1).
18. Ficker L, Vafidis G, While A, Leaver P. Long-term follow-up of a prospective trial of argon laser photocoagulation in the treatment of central serous retinopathy. Br J Ophthalmol 1988;72(11):829-834.

19. Chhablani J, Rani PK, Mathai A, Jalali S, Kozak I. Navigated focal laser photocoagulation for central serous chorioretinopathy. Clin Ophthalmol 2014 Aug 18;8:1543-1547.

20. Ambiya V, Khodani M, Goud A, et al. Early focal laser photocoagulation in acute central serous chorioretinopathy: a prospective, randomized study. Ophthalmic Surg Lasers Imaging Retina 2017 Jul 1;48(7):564-571.

21. Abouammoh MA. Advances in the treatment of central serous chorioretinopathy. Saudi J Ophthalmol 2015;29(4):278-286.

22. Ruiz-del-Tiempo MP, Calvo P, Ferreras A, Leciñena J, Pablo L, Ruiz-Moreno O. Anatomical retinal changes after photodynamic therapy in chronic central serous chorioretinopathy. J Ophthalmology 2018;2018:1-4.

23. Daruich A, Matet A, Dirani A, et al. Central serous chorioretinopathy: Recent findings and new physiopathology hypothesis. Prog Retin Eye Res 2015 Sep;48:82-118.

24. Zhao M, Bousquet E, Valamanesh F, et al. Differential regulations of AQP4 and Kir4.1 by triamcinolone acetonide and dexamethasone in the healthy and inflamed retina. Invest Opthalmol Vis Sci 2011;52(9):6340.

25. Wong KH, Lau KP, Chhablani J, Tao Y, Li Q, Wong IY. Central serous chorioretinopathy: what we have learnt so far. Acta Ophthalmol 2016;94(4):321-325.

26. Daruich A, Matet A, Marchionno L, et al. Acute central serous chorioretinopathy: factors influencing episode duration. Retina 2017;37(10):1905-1915.

27. Do JL, Olmos de Koo LC, Ameri H. Atypical chronic central serous chorioretinopathy with cystoid macular edema: Therapeutic response to medical and laser therapy. J Curr Ophthalmol 2017;29(2):133-135.

28. Zucchiatti I, Sacconi R, Parravano MC, et al. Eplerenone versus observation in the treatment of acute central serous chorioretinopathy: a retrospective controlled study. Ophthalmol Ther 2018 Jun;7(1):109-118.

29. Nicholson B, Noble J, Forooghian F, Meyerle C. Central serous chorioretinopathy: update on pathophysiology and treatment. Surv Ophthalmol 2013;58(2):103-126.

30. Caccavale A, Romanazzi F, Imparato M, Negri A, Morano A, Ferentini F. Low-dose aspirin as treatment for central serous chorioretinopathy. Clin Ophthalmol 2010;4:899-903.

31. Chung Y-R, Seo EJ, Lew HM, Lee KH. Lack of positive effect of intravitreal bevacizumab in central serous chorioretinopathy: metaanalysis and review. Eye 2013;27(12):1339-1346. 